\title{
Effectiveness of treatment with nebulized colistin in patients with COPD
}

\author{
This article was published in the following Dove Press journal: \\ International Journal of COPD \\ 5 October 2017 \\ Number of times this article has been viewed
}

\author{
Nuria Bruguera-Avila ${ }^{1-3}$ \\ Alicia Marin ${ }^{3-5}$ \\ Ignasi Garcia-Olive ${ }^{3-5}$ \\ Joaquim Radua ${ }^{6-8}$ \\ Cristina Prat ${ }^{4,9,10}$ \\ Montserrat $\mathrm{Gil}^{3}$ \\ Juan Ruiz-Manzano ${ }^{2-5}$
}

'Department of Medicine, Hospital Sant Jaume de Calella, Calella, Barcelona, Spain; ${ }^{2}$ Department of Medicine, Universitat Autònoma de Barcelona, Bellatera, Spain: ${ }^{3}$ Department of Pulmonary Medicine, Hospital Universitari Germans Trias i Pujol, Carretera del Canyet sn, Badalona, Barcelona, Spain; ${ }^{4}$ Ciber de Enfermedades Respiratorias, Instituto de Salud Carlos III, Madrid, Spain; ${ }^{5}$ Fundació Institut d'Investigació en Ciències de la Salut Germans Trias i Pujol, Carretera del Canyet sn, Badalona, Barcelona, Spain; ${ }^{6}$ Department of Statistics, FIDMAG Germanes Hospitalaries Research Unit, Sant Boi de Llobregat, Barcelona, Spain; ${ }^{7}$ CiberSam - Ciber de Salud Mental, Madrid, Spain; ${ }^{8}$ Centre for Psychiatry Research, Department of Clinical Neuroscience, Karolinska Institutet, Stockholm, Sweden; ' Department of Microbiology, Hospital Universitari Germans Trias i Pujol, Carretera del Canyet sn, Badalona, Barcelona, Spain; ${ }^{10}$ Department of Genetics and Microbiology of Universitat Autònoma de Barcelona, Bellatera, Spain

Correspondence: Alicia Marin Respiratory Service, Hospital Universitari Germans Trias i Pujol, Carretera de Canyet sn, 08916 Badalona, Barcelona, Spain Tel +34934978920

Fax +34934978722

Email amarin.germanstrias@gencat.cat
Objectives: To analyze whether the introduction of nebulized colistin in patients with chronic obstructive pulmonary disease (COPD) and infection with Pseudomonas aeruginosa (PA) is associated with a decrease of the number and duration of severe exacerbations.

Materials and methods: Thirty six patients with COPD and infection with PA treated with nebulized colistin attending a day hospital during a 5-year (January 2010-December 2014) period were prospectively included. Repeated-measures $t$-tests were used to assess whether the introduction of colistin was associated with changes in the number of exacerbations or the length of the hospitalizations, comparing for each patient the year prior to the introduction of colistin with the year after.

Results: After the introduction of colistin, the number of admissions decreased from 2.0 to 0.9 per individual year $(P=0.0007)$, and hospitalizations were shorter ( 23.3 vs 10.9 days, $P=0.00005)$. These results persisted when patients with and without bronchiectasis or with and without persistence of Pseudomonas were separately analyzed. No pre-post differences were detected in the number of exacerbations not requiring admission.

Conclusion: Nebulized colistin seems associated with a strong decrease in the number and duration of hospitalizations due to exacerbation in patients with COPD and infection with PA. Clinical trials with a larger number of patients are needed in order to confirm these results.

Keywords: bronchiectasis, colistin, COPD, nebulized antibiotics

\section{Introduction}

Patients with chronic obstructive pulmonary disease (COPD) often suffer from acute exacerbations of their disease, which have a negative impact on quality of life, ${ }^{1}$ accelerate disease progression, and cause increased health care utilization and costs, ${ }^{2,3}$ especially in those requiring hospitalization. Evidence suggests that about $50 \%$ of these exacerbations are attributable to bacteria, ${ }^{4,5}$ with the most frequently potential pathogenic microorganisms (PPMs) isolated being Haemophilus influenzae, Streptococcus pneumoniae, and Moraxella catharralis, ${ }^{6}$ while Pseudomonas aeruginosa (PA) is usually isolated in subjects with worse forced expiratory volume in 1 second $\left(\mathrm{FEV}_{1}\right)$ values with an estimated prevalence between $8 \%-13 \%{ }^{7,8}$ The presence of PA infection in COPD has also been related to risk factors such as previous hospital admissions, use of oral corticosteroids or antibiotics, and the presence of bronchiectasis as well as its extent. ${ }^{9-12}$ It has been demonstrated that a higher mortality after hospital discharge is observed in patients with COPD and PA isolation in sputum culture independently of other relevant predictors such as age, comorbidity, or BODE (body mass index, airflow obstruction, dyspnea, and exercise capacity) index. ${ }^{13}$ Recent studies demonstrate that PA has different features in COPD patients, with the most frequent pattern being sporadic or intermittent infection. ${ }^{14,15}$ However, studies based on pulsed-field 
gel electrophoresis showed a pattern of chronic infection with persistent clones similar to cystic fibrosis (CF) in up to $10 \%$ of patients, most of them in severe COPD stages. ${ }^{12,16,17}$ Contrary to what occurs in $\mathrm{CF}$, in which the use of long-term nebulized antibiotics is accepted, for the treatment of PA chronic infection, the management of patients with repeated isolates of this PPM is not well established. In this study, we investigate the effectiveness of long-term nebulized colistin in reducing exacerbation frequency and hospital admissions in COPD patients with PA chronic infection.

\section{Methods}

\section{Study subjects}

COPD patients (defined by a $\mathrm{FEV}_{1}$ /forced vital capacity [FVC] below 0.7 in patients with at least 10 pack-years of tobacco use according to the Global Initiative for Chronic Obstructive Lung Disease criteria) with an $\mathrm{FEV}_{1}$ postbronchodilatador $\leq 50 \%$ of the reference value and frequent exacerbations (two or more exacerbations in the previous year) who are seen at the respiratory day care unit of Hospital Universitari Germans Trias i Pujol for scheduled and exacerbation visits. ${ }^{18}$ Those COPD patients with chronic bronchial colonization by PA (at least three consecutive sputum cultures during a 6-month period of stability), or intermittent bronchial colonization (alternating positive cultures with negative cultures at least one month apart) are treated with nebulized colistin. The eradication protocol when PA was first isolated included at least one treatment of 15-21 days of antibiotic chosen according to antibiogram. The use of longterm macrolides was not included in the protocol center, and none of these patients followed this treatment.

The study prospectively included 36 consecutive subjects with COPD treated with nebulized colistin attending a day hospital during a 5-year period (January 2010December 2014). Patients with asthma, malignancy, or unstable heart disease were excluded, as were patients with a main diagnosis of bronchiectasis.

\section{Study design}

The primary endpoint of the study was the number of severe exacerbations requiring hospital admissions and their duration and, secondarily, the number of mild exacerbations not requiring hospitalization, comparing the year prior to starting nebulized colistin with the year after the beginning of the treatment. Data about COPD exacerbations was thoroughly investigated on many fronts, including a review of hospital and outpatient medical records, computerized databases, and, when necessary, telephone contact with the patient or his/her relatives or primary care physician. COPD exacerbation was defined as an acute worsening of respiratory symptoms resulting in additional therapy, it was classified as moderate when the prescription of courses of antibiotics and/or oral corticosteroids was required, and it was considered severe when patient required hospitalization or visited the emergency room. ${ }^{18}$

At baseline, smoking history, severity of the disease, airflow obstruction, comorbidities (Charlson Comorbidity Index), use of long-term home oxygen therapy, use of ongoing inhaled therapy, and short-term treatments with antibiotics and corticosteroids due to respiratory causes in the previous year were recorded. The diagnosis of bronchiectasis was based on chest high-resolution computed tomography scan. High-resolution images were obtained in full inspiration at $1 \mathrm{~mm}$ collimation and $10 \mathrm{~mm}$ intervals from the apex to the base of the lungs. The diagnosis of bronchiectasis was based on criteria published by Naidich et al. ${ }^{19}$

\section{Nebulization technique}

One million international units of colistin in $1 \mathrm{~mL} 0.45 \%$ saline was administered through the I-neb adaptive aerosol delivery device (Philips Respironics, Chichester, UK). The I-neb is a unique device that monitors the time and peak flow of the first three breaths during nebulization and then pulses aerosol at the start of the inspiratory phase to optimize drug delivery. The first dose was administered under supervision, and 2 weeks after the start of treatment a review of the nebulization technique was made. Further, the drug was self-administered twice a day. Adherence was evaluated using dispensing data of colistin from the hospital pharmacy and directly from the patient at every 2 month scheduled visit. We did not evaluate direct compliance data using the specific software for use with the I-neb AAD System. Only patients who completed at least 3 months of treatment were included in the analysis.

\section{Bacteriological assessment}

Sputum samples were collected from all patients when able to produce it during the treatment period at every 2 month scheduled visit and for each acute exacerbations of COPD before antibiotic administration, and these samples were processed locally for Gram stain and semiquantitative bacteriological culture. Identification and antibiogram were performed on all bacterial isolates following standardized microbiological protocols. ${ }^{20}$ Colistin was routinely tested in all Gram-negative bacteria by disk diffusion in enterobacteriaceae and by VITEK (biomerieux, Marcy-l'Étoile, France) in nonfermenters. Resistance was only reported in bacteria known as constitutively 
resistant. Eradication of PA was defined as one year of follow-up without PA isolation in good quality sputum.

\section{Statistical analysis}

Repeated-measures $t$-tests were used to assess whether there were statistically significantly pre-post differences in the number of exacerbations not requiring admission, the number of admissions, and the length of the admissions. These differences had been previously checked to be approximately normally distributed (Shapiro-Wilk test $P$-values $>0.367$ ). The Student's $t$-tests were separately conducted for the overall sample and for the subsamples of patients with and without bronchiectasis. Additionally, linear regressions on the differences were used to assess whether the effects of treatment might depend on $\mathrm{COPD}$ severity, on $\mathrm{FEV}_{1} \%$, and on the number of lobes with bronchiectasis (in patients with bronchiectasis).

\section{Ethical considerations}

The research protocol was approved by the regional ethics committee (Ethics Committee for Clinical Research of the Hospital Germans Trias i Pujol). All patients provided written informed consent to be included in this research.

\section{Results}

Thirty-six patients were included in the analysis. Baseline characteristics of these patients can be seen in Table 1 . Bronchiectasis was detected on high-resolution computed tomography in 27 of the 36 patients $(75 \%)$. These patients did not show differences in baseline characteristics when compared with those without bronchiectasis, except for a lower $\mathrm{FEV}_{1}$ postbronchodilation (Table 2). No multidrug-resistant PA strains were observed, although in some cases there was resistance to quinolones. The average treatment duration was $10.7 \pm 4.2$ months. A total of six patients discontinued treatment (16.6\%). Four patients reported bronchospasm (11.1\%) and three of them discontinued for this reason $(8.4 \%)$. There were two deaths (5.5\%), both after the follow-up year, and so these were considered unlikely related to the treatment (1 COPD exacerbation, 1 pneumonia). One patient stopped treatment because of their own decision (2.8\%). Eradication of PA was observed in 14 patients (38.9\%). No differences in the culture results during follow-up were found between patients with and without bronchiectasis (Table 3). Resistance to colistin was only reported in bacteria known as constitutively resistant.

The number of admissions was found to decrease from 2.0 before the treatment to 0.9 after the treatment $(t=3.7$, $d f=35, P=0.0007)$, and these were shorter (23.3 days before the treatment, 10.9 days after the treatment, $t=4.7, d f=35$,
Table I Baseline characteristics of COPD patients chronically colonized with PA

\begin{tabular}{|c|c|}
\hline Characteristics & Results \\
\hline Subjects, $n$ & 36 \\
\hline Gender male, $\mathrm{n}(\%)$ & $36(100)$ \\
\hline Age (years), m (SD) & $72.7(2.1)$ \\
\hline Current smoking, n (\%) & $0(0)$ \\
\hline Pack-years, m (SD) & $4 \mid(4.5)$ \\
\hline FEV, post-BD, (L), m (SD) & $1.15(0.3)$ \\
\hline FEV , post-BD, (\% predicted), $m(S D)$ & $38(2)$ \\
\hline Charlson Comorbidity Index, m (SD) & $3.08(1.54)$ \\
\hline \multicolumn{2}{|l|}{ Daily treatments, n (\%) } \\
\hline LAMA & $34(94.4)$ \\
\hline LABA & $34(94.4)$ \\
\hline ICS & $33(91.7)$ \\
\hline LTOT & $15(4 \mid .7)$ \\
\hline Hospitalizations due to AECOPD/past year, m (SD) & $2(0.27)$ \\
\hline Days of hospital stay/past year, m (SD) & $23.3(3.4)$ \\
\hline Ambulatory AECOPD/past year, m (SD) & I.7 (0.26) \\
\hline \multicolumn{2}{|l|}{ CT scan findings } \\
\hline Patients with bronchiectasis, $\mathrm{n}(\%)$ & $27(75)$ \\
\hline \multicolumn{2}{|l|}{ Type of bronchiectasis, n (\%) } \\
\hline Cylindrical & $19(70.4)$ \\
\hline Cystic & $5(18.5)$ \\
\hline Both & $3(I I . I)$ \\
\hline \multicolumn{2}{|l|}{ Number of affected lobes, $n$ (\%) } \\
\hline 1 & $4(I I .1)$ \\
\hline 2 & II (30.5) \\
\hline 3 & $10(27.8)$ \\
\hline 4 & I (2.8) \\
\hline 5 & I (2.8) \\
\hline
\end{tabular}

Abbreviations: COPD, chronic obstructive pulmonary disease; PA, Pseudomonas aeruginosa; $S D$, standard deviation; $\mathrm{FEV}_{1}$, forced expiratory volume in one second; $B D$, bronchodilator; LAMA, long-acting anti-muscarinic; LABA, long-acting $\beta$-agonist; ICS, inhaled corticosteroids; LTOT, long-term oxygen therapy; AECOPD, acute exacerbation of chronic obstructive pulmonary disease; $\mathrm{CT}$, computed tomography.

$P=0.00005)$. These results persisted when patients with and without bronchiectasis were separately analyzed. Specifically, the number of admissions decreased from 2.0 to 1.0 in patients with bronchiectasis $(t=3.2, d f=26, P=0.003)$ and from 2.0 to 0.8 in patients without bronchiectasis $(t=1.8, d f=8$, $P=0.111$ ). Similarly, the length of the admissions decreased from 24.1 to 12.0 days in patients with bronchiectasis $(t=3.8$, $d f=26, P=0.0008)$ and from 20.8 to 7.8 days in patients without bronchiectasis $(t=2.6, d f=8, P=0.031$ ). No pre-post differences were detected in the number of moderate exacerbations not requiring admission (Table 4).

The effects of treatment were not found to depend on COPD severity, on $\mathrm{FEV}_{1} \%$, or on the number of lobes with bronchiectasis.

\section{Discussion}

This study shows that the use of long-term nebulized colistin is associated with a decrease in the number and length of 
Table 2 Baseline characteristics of COPD patients chronically colonized with PA according to the presence or absence of bronchiectasis

\begin{tabular}{|c|c|c|c|}
\hline Characteristics & $\begin{array}{l}\text { With } \\
\text { bronchiectasis }\end{array}$ & $\begin{array}{l}\text { Without } \\
\text { bronchiectasis }\end{array}$ & $P$-value \\
\hline Subjects, $n$ & 27 & 9 & - \\
\hline Gender male, $\mathrm{n}(\%)$ & $27(100)$ & $9(100)$ & I \\
\hline Age (years), m (SD) & $72.2(1.8)$ & $74.4(2.3)$ & 0.53 \\
\hline Current smoking, n (\%) & $0(0)$ & $0(0)$ & I \\
\hline Pack-years, m (SD) & $37.4(4.3)$ & $55.0(34.4)$ & 0.08 \\
\hline FEV, post-BD, (L), m (SD) & $\mathrm{I} .06(0.07)$ & $1.43(0.21)$ & 0.04 \\
\hline FEV , post-BD, (\% predicted), $\mathrm{m}(\mathrm{SD})$ & $36.4(2.2)$ & $43.5(4.3)$ & 0.13 \\
\hline Hospitalizations due to AECOPD/previous year, m (SD) & $2(0.3)$ & $2(0.8)$ & I \\
\hline Days of hospital stay/past year, m (SD) & $24.1(4.0)$ & $20.8(6.8)$ & 0.68 \\
\hline Ambulatory AECOPD/previous year, m (SD) & $1.6(0.3)$ & $2.2(0.7)$ & 0.28 \\
\hline
\end{tabular}

Abbreviations: COPD, chronic obstructive pulmonary disease; PA, Pseudomonas aeruginosa; SD, standard deviation; FEV , forced expiratory volume in one second; $\mathrm{BD}$, bronchodilator; $\mathrm{AECOPD}$, acute exacerbation of chronic obstructive pulmonary disease.

hospitalizations due to exacerbation in patients with COPD and chronic infection with PA independently of the presence or not of bronchiectasis.

The use of different regimes of long-term antibiotic prophylaxis has been proposed in COPD patients with frequent bacterial exacerbations, especially those with chronic bronchial infection, as a strategy to prevent exacerbations by reducing the bacterial load and/or by preventing the acquisition of new strains. ${ }^{21}$ The most common regimens accepted are continuous administration of oral macrolides and intermittent oral quinolones in selected patients and under strict clinical control. ${ }^{22}$ Long-term inhaled antibiotics are an attractive alternative in patients with COPD with frequent exacerbations. The rationale for nebulized antibiotic therapy is to maximize drug delivery to the respiratory airways and limit the potential for systemic side effects, and this has demonstrated its benefit in the treatment of respiratory chronic infection in CF bronchiectasis. ${ }^{23}$ Although CF remains the only pulmonary condition in which inhaled antibiotics have received US Food and Drug Administration and European Medicines Agency approval, there is a growing interest in the used of this therapy in other conditions outside of $\mathrm{CF}$, such as non-CF bronchiectasis (NCFB) and COPD.

Table 3 Microbiological evolution during the follow-up year according to the presence or absence of bronchiectasis

\begin{tabular}{llll}
\hline Microorganisms & $\begin{array}{l}\text { With } \\
\text { bronchiectasis } \\
\mathbf{n = 2 7}\end{array}$ & $\begin{array}{l}\text { Without } \\
\text { bronchiectasis } \\
\mathbf{n = 9}\end{array}$ & P-value \\
\hline PA, n (\%) & $17(62)$ & $5(55)$ & 0.17 \\
$\begin{array}{l}\text { Nonfermenting } \\
\text { Gram-negative, }{ }^{\text {a }} \text { n (\%) }\end{array}$ & $2(7.4)$ & 0 & 0.30 \\
Fungal, ${ }^{\mathrm{b}}$ (\%) & $4(15)$ & 0 & 0.13 \\
\hline
\end{tabular}

Notes: ${ }^{N}$ Nonfermenting Gram-negative: Acinetobacter baumannii, Achromobacter xylosoxidans, Burkholderia cepacia, Stenotrophomonas maltophilia. 'Fungal: Aspergillus fumigatus.

Abbreviation: PA, Pseudomonas aeruginosa.
These respiratory tract conditions can present characteristics of persistent or recurrent infection of the airway by bacteria such as H. influenzae or PA that contribute to the chronic cycle of infection and inflammation. ${ }^{24,25} \mathrm{PA}$ is found in approximately $25 \%$ of patients with NCFB, and it has been associated with more frequent exacerbations, faster disease progression, and increased mortality. ${ }^{11,24,26}$ Because of the worse clinical outcomes associated with chronic infection with PA, the British Thoracic Society guidelines on bronchiectasis recommend the prescription of nebulized antibiotics in such patients if they have a high infection frequency. ${ }^{27}$ Such a recommendation is supported by several studies evaluating different nebulized antibiotics. A recent meta-analysis performed to determine the efficacy and safety of long-term inhaled antibiotics for patients with NCFB included nine studies, 5 on inhaled aminoglycoside, 2 on inhaled ciprofloxacin, and 1 on inhaled colistin, and demonstrated the reduction of sputum bacterial density and the increase of eradication of PA. Moreover, nebulized therapy decreased risk of exacerbations but failed to improve $\mathrm{FEV}_{1}$ and St. George's Respiratory Questionnaire (SGRQ) score..$^{28}$

To date, there have been only few published reports examining the effects of inhaled antibiotics on health outcomes in COPD. Dal Negro et $\mathrm{al}^{29}$ examined the effect of nebulized tobramycin twice a day for 14 days in 13 patients with severe COPD colonized by multiresistant PA. The authors found a reduction in local inflammation and bacterial load (and even eradication in some patients), and finally a $42 \%$ reduction in severe exacerbations after 6 months of treatment. Steinfort et $\mathrm{al}^{30}$ studied the effect of nebulized colistin during 18 months in patients chronically infected by Gramnegative bacteria (14 idiopathic bronchiectasis, 4 severe COPD). The authors observed significantly slower decline of $\mathrm{FEV}_{1}$ and $\mathrm{FVC}$ and an improvement in patient-reported quality of life. ${ }^{30}$ In a pilot study conducted at a day hospital 
Table 4 Results and comparison between pre- and posttreatment outcomes according to the presence or absence of bronchiectasis

\begin{tabular}{|c|c|c|c|c|c|c|c|c|c|c|c|c|}
\hline \multirow[t]{3}{*}{ Outcome variables } & \multirow{2}{*}{\multicolumn{4}{|c|}{ Whole group $n=36$}} & \multicolumn{8}{|c|}{ According to presence of bronchiectasis } \\
\hline & & & & & \multicolumn{4}{|c|}{ With bronchiectasis $n=27$} & \multicolumn{4}{|c|}{ Without bronchiectasis $\mathrm{n}=9$} \\
\hline & $\begin{array}{l}\text { Pre }^{\mathrm{a} / \text { post }^{\mathrm{b}}} \\
\text { colistin }\end{array}$ & Diff (SD) & $t_{35}$ & $P$-value & $\begin{array}{l}\text { Pre }^{a} / \text { post }^{\mathrm{b}} \\
\text { colistin }\end{array}$ & Diff (SD) & $t_{26}$ & $P$-value & $\begin{array}{l}\text { Pre }^{\mathrm{a} / \text { post }^{\mathrm{b}}} \\
\text { colistin }\end{array}$ & Diff (SD) & $t_{8}$ & $P$-value \\
\hline $\begin{array}{l}\text { Hospitalizations due to } \\
\text { AECOPD, } m(S D)\end{array}$ & $2 / 0.9$ & $-1.1(1.7)$ & 3.7 & 0.000 & $2 / 1$ & $-1(1.6)$ & 3.2 & 0.003 & $2 / 0.8$ & $-1.2(2.0)$ & 1.8 & 0.11 \\
\hline Days of hospital stay, m (SD) & $23 / 11$ & $-12(15.9)$ & 4.6 & 0.000 & $24 / 12$ & $-12(16.6)$ & 2.6 & 0.000 & $21 / 8$ & $-13(14.9)$ & 2.6 & 0.03 \\
\hline Ambulatory AECOPD, m (SD) & $1.7 / 1.5$ & $-0.2(1.9)$ & 0.7 & 0.50 & $1.5 / 1.2$ & $-0.3(1.9)$ & 0.9 & 0.61 & $2.2 / 2.3$ & $-0.1(2.0)$ & 0.2 & 0.87 \\
\hline
\end{tabular}

Notes: ${ }^{a}$ Data from the previous year to start colistin. ${ }^{b}$ Data at 12 months after the start of colistin.

Abbreviations: SD, standard deviation; AECOPD, acute exacerbation of chronic obstructive pulmonary disease; Diff, difference.

unit specializing in patients with severe and very severe COPD and a high risk of exacerbations by PA, the use of nebulized colistin was associated with a significant reduction in the number of exacerbations and emergency visits during a year of follow-up. ${ }^{31}$

Although those studies have many limitations, including their open-label design and their small size, all of them suggest a therapeutic role for inhaled antibiotics in COPD patients. However, a phase II study investigating the efficacy of MP-376 (levofloxacin inhaled solution) in COPD patients at high risk for exacerbations failed to demonstrate a significant reduction in the exacerbation rate or an increase in the time to the next exacerbation compared with placebo. ${ }^{32}$ Probably the treatment duration employed in this study ( 5 days every 28 days for $9-12$ cycles) may have been suboptimal in a population of patients with chronic infection. ${ }^{33}$

Our study is the first one assessing the effect of longterm inhaled antibiotic in an extended cohort of patients with severe COPD patients who present with repeated isolates of PA despite having been treated at least once with antibiotic therapy with eradicating intention based on antibiogram. These patients presented during the previous year frequent exacerbations and included those requiring hospitalization with a long average stay. After at least 6 months of treatment with nebulized colistin the number and length of hospital admissions for COPD exacerbation was reduced significantly, although no differences were detected in the number of exacerbations not requiring admission. These findings are consistent with previous reports in NCFB patients. ${ }^{34-36}$ The effect of treatment was observed regardless of the presence of bronchiectasis, or of the number of lobes affected. In our cohort, bronchiectasis was present in up to $75 \%$ of the patients, with cylindrical being the more frequent type reported affecting 2 or 3 lobes in most of the cases. This incidence of bronchiectasis is a little higher than that reported by other authors probably due to the selected population..$^{10-12}$ The relationship between the presence of bronchiectasis and PA infection has been evaluated with conflicting results. Martínez-García et al, ${ }^{11}$ in a study assessing risk factors for bronchiectasis in COPD patients, found that bronchial colonization by PPMs was associated with the presence of bronchiectasis, but the presence of PA, in the form of either a single isolate or chronic colonization, was not. The same results were reported by García-Vidal et al. ${ }^{10}$ Gallego et al, ${ }^{12}$ in one study evaluating the prevalence and risk factors for PA in severe COPD patients, observed that the isolation of PA was significantly associated with the extent of bronchiectasis. However, the presence of chronic infection by PA, a situation that occurred in up to $10 \%$ of subjects, was unrelated to the presence of bronchiectasis. These data suggest that although chronic bronchial infection in COPD patients is very frequently associated with bronchiectasis, it is not the only determining factor.

In our study, nebulized colistin has been generally well tolerated, and only four patients (11\%) presented adverse effects such as wheezing and bronchospasm, and only three of these patients discontinued treatment. In a previous metaanalysis where clinical trials with inhaled antibiotics were analyzed, a higher incidence of wheezing and bronchospasm was described in patients receiving treatment, but this was similar to the placebo group and therefore resulted in low withdrawal due to these adverse events. ${ }^{28}$ For this reason, patients are often routinely instructed to administer bronchodilators before the dose of nebulized colistin.

We observed eradication of PA during one year of follow-up in $40 \%$ of cases, and no development of resistance to colistin was recorded. There are several studies that support the efficacy of nebulized colistin to eradicate PA in sputum, reaching eradication rates between $20 \%$ and $60 \%{ }^{30,35,37}$ Nevertheless, our findings suggest that clinical benefits of nebulized therapy with colistin are not necessarily related to eradication of $\mathrm{PA}$, but to the decrease in the bacterial load in the sputum of these patients. 
There are several limitations to this study. First, this is a retrospective study. Second, the sample size was small. And finally, there was no control group to enable comparison of the results. However, our results open the possibility to treat the negative clinical condition of chronic bronchial infection by PA in patients with COPD, which is cause of severe exacerbations and has a very negative impact on the evolution of the disease, that clinicians find difficult to handle.

\section{Conclusion}

In conclusion, this study shows that the use of nebulized colistin is associated with a decrease in the number and length of hospitalizations due to exacerbation in patients with COPD and infection with PA. These findings occurred in patients with and without bronchiectasis. Clinical trials in this population with a larger number of patients are desirable in order to confirm these results. The effect that long-term treatment may have on the selection of emerging pathogens should also be assessed. And finally, economic studies should be done by comparing the economic impact between expenditure arising from such long-term treatment with the expenses generated by exacerbations and hospital admissions.

\section{Disclosure}

The authors do not have any financial or personal relationships with people or organizations that could inappropriately influence their work in the present article. The authors report no conflicts of interest in this work.

\section{References}

1. Kelly JL, Bamsey O, Smith C, et al. Health status assessment in routine clinical practice: the chronic obstructive pulmonary disease assessment test score in outpatients. Respiration. 2012;84(3):193-199.

2. Donaldson GC, Seemungal TA, Bhowmik A, Wedzicha JA. Relationship between exacerbation frequency and lung function decline in chronic obstructive pulmonary disease. Thorax. 2002;57(10):847-852.

3. Miravitlles M, García-Polo C, Domenech A, Villegas G, Conget F, de la Roza C. Clinical outcomes and cost analysis of exacerbations in chronic obstructive pulmonary disease. Lung. 2013;191(5):523-530.

4. Sethi S. Infectious etiology of acute exacerbations of chronic bronchitis. Chest. 2000;117:380S-385S.

5. Bafadhel M, McKenna S, Terry S, et al. Acute exacerbations of chronic obstructive pulmonary disease. Identification of biologic clusters and their biomarkers. Am J Respir Crit Care Med. 2011;184:662-671.

6. Rosell A, Monso E, Soler N, et al. Microbiologic determinants of exacerbation in chronic obstructive pulmonary disease. Arch Intern Med. 2005; 165:891-897.

7. Eller J, Ede A, Schaberg T, Niederman M, Mauch H, Lode H. Infective exacerbation of chronic bronchitis. Relation between bacteriologic etiology and lung function. Chest. 1998;113:1542-1548.

8. Lieberman D, Lieberman D. Pseudomonal infections in patients with COPD: epidemiology and management. Am J Respir Med. 2003;2(6): 459-468.

9. Lode $\mathrm{H}$, Allewelt $\mathrm{M}$, Balk $\mathrm{S}$, et al. A prediction model for bacterial etiology in Acute Exacerbations of COPD. Infection. 2007;35:143-149.
10. García-Vidal C, Almagro P, Romaní V, et al. Pseudomonas aeruginosa in patients hospitalised for COPD exacerbation: a prospective study. Eur Respir J. 2009;34:1072-1078.

11. Martínez-García MA, Soler-Cataluña JJ, Donat Y, et al. Factors associated with bronchiectasis in patients with COPD. Chest. 2011;140: $1130-1137$.

12. Gallego M, Pomares X, Espasa M, et al. Pseudomonas aeruginosa isolates in severe chronic obstructive pulmonary disease: characterization and risk factors. BMC Pulm Med. 2014;14:103.

13. Almagro P, Salvadó M, Garcia-Vidal C, et al. Pseudomonas aeruginosa and mortality after hospital admission for chronic obstructive pulmonary disease. Respiration. 2012;84(1):36-43.

14. Murphy TF. Pseudomonas aeruginosa in adults with chronic obstructive pulmonary disease. Curr Opin Pulm Med. 2009;15(2):138-142.

15. Rakhimova E, Wiehlmann L, Brauer AL, Sethi S, Murphy TF, Tümmler B. Pseudomonas aeruginosa population biology in chronic obstructive pulmonary disease. J Infect Dis. 2009;200:1928-1935.

16. Martínez-Solano L, Macia MD, Fajardo A, Oliver A, MartínezJL. Chronic Pseudomonas aeruginosa infection in chronic obstructive pulmonary disease. Clin Infect Dis. 2008;47:1526-1533.

17. Valderrey AD, Pozuelo MJ, Jiménez PA, Maciá MD, Oliver A, Rotger R. Chronic colonization by Pseudomonas aeruginosa of patients with obstructive lung disease: cystic fibrosis, bronchiectasis and chronic obstructive pulmonary disease. Diagn Microbiol Infect Dis. 2010; 68:20-27.

18. From the Global Strategy for the Diagnosis, Management and Prevention of COPD, Global Initiative for Chronic Obstructive Lung Disease (GOLD); 2017. Available from: http://goldcopd.org. Accessed February 4, 2017.

19. Naidich DP, McCauley DI, Khouri NF, Stitik FP, Siegelman SS. Computed tomography of bronchiectasis. J Comput Assist Tomogr. 1982; 6:437-444.

20. Clinical and Laboratory Standards Institute. Performance Standards for Antimicrobial Susceptibility Testing; Twentieth Informational Supplement. Wayne, PA: Clinical and Laboratory Standards Institute; 2010.

21. Wilson R, Sethi S, Anzueto A, Miravitlles M. Antibiotics for treatment and prevention of exacerbations of chronic obstructive pulmonary disease. J Infect. 2013;67(6):497-515.

22. Miravitlles M, Soler-Cataluña JJ, Calle M, et al. Spanish Society of Pulmonology and Thoracic Surgery. Spanish COPD Guidelines (GesEPOC): pharmacological treatment of stable COPD. Spanish Society of Pulmonology and Thoracic Surgery. Arch Bronconeumol. 2012;48:247-257.

23. Fiel SB. Aerosolized antibiotics in cystic fibrosis: an update. Fiel SB. Expert Rev Respir Med. 2014;8(3):305-314.

24. Angrill J, Agusti C, de Celis R, et al. Bacterial colonisation in patients with bronchiectasis: microbiological pattern and risk factors. Thorax. 2002;57:15-19.

25. Sethi S, Murphy TF. Infection in the pathogenesis and course of chronic obstructive pulmonary disease. $N$ Engl J Med. 2008;359:2355-2365.

26. Loebinger MR, Wells AU, Hansell DM, et al. Mortality in bronchiectasis: a long-term study assessing the factors influencing survival. Eur Respir J. 2009;34:843-849.

27. Pasteur MC, Bilton D, Hill AT; British Thoracic Society Bronchiectasis Non-CF Guideline Group. British Thoracic Society guideline for non-CF bronchiectasis. Thorax. 2010;65(Suppl 1):i1-i58.

28. Yang JW, Fan LC, Lu HW, Miao XY, Mao B, Xu JF. Efficacy and safety of long-term inhaled antibiotic for patients with noncystic fibrosis bronchiectasis: a meta-analysis. Clin Respir J. 2016;10(6):731-739.

29. Dal Negro R, Micheletto C, Tognella S, et al. Tobramycin Nebulizer Solution in severe COPD patients colonized with Pseudomonas aeruginosa: effects on bronchial inflammation. Adv Ther. 2008;25: $1019-1030$

30. Steinfort DP, Steinfort C. Effect of long-term nebulised colistinon lung function and quality of life in patients with chronic bronchial sepsis. Intern Med J. 2007;37:495-498. 
31. Manuel MJ, Muñoz-Ezquerre M, López-Sánchez M, et al. Adherencia y efectividad en el control de exacerbaciones en pacientes con EPOC grave bajo el tratamiento con colimicina inhalada (Promixin ${ }^{\mathbb{R}}$ ) en dispositivo I-neb. [Adherence and effectiveness in the control of exacerbations in patients with severe COPD under treatment with inhaled colimicin (Promixin ${ }^{\circledR}$ ) in an I-neb device]. Arch Bronconeum. 46th National Congress Spanish Society of Pulmonology and Thoracic Surgery; 2012. [Abstract]. Spanish.

32. Sethi S, Rennard SI, Miravitlles M, et al. A Phase 2 study to evaluate the safety, tolerability and efficacy of levofloxacin inhalation solution (MP-376) administered for 5 days every 28 days to prevent acute exacerbations in high risk COPD patients. Am J Respir Crit Care Med 2012;185:A3037.

33. Azoicai D, Antoniu S. MP-376 (Aeroquin) for chronic Pseudomonas aeruginosa infections. Expert Opin Investig Drugs. 2013;22: $267 \mathrm{e} 76$.
34. Drobnic ME, Suñé P, Montoro JB, Ferrer A, Orriols R. Inhaled tobramycin in non-cystic fibrosis patients with bronchiectasis and chronic bronchial infection with Pseudomonas aeruginosa. Ann Pharmacother. 2005;39:39-44.

35. Dhar R, Anwar GA, Bourke SC, et al. Efficacy of nebulised colomycin in patients with non-cystic fibrosis bronchiectasis colonised with Pseudomonas aeruginosa. Thorax. 2010;65:553.

36. Murray MP, Govan JR, Doherty CJ, et al. A randomized controlled trial of nebulised gentamicin in non-cystic fibrosis bronchiectasis. Am J Respir Crit Care Med. 2011;183:491-499.

37. Harun NS, Sanders K, Stuart Steinfort C. Long-term nebulised colistin aids sputum clearance of pseudomonas aeruginosa in adult noncystic fibrosis bronchiectasis. Am J Respir Crit Care Med. 2011;183: A4865.

\section{Publish your work in this journal}

The International Journal of COPD is an international, peer-reviewed journal of therapeutics and pharmacology focusing on concise rapid reporting of clinical studies and reviews in COPD. Special focus is given to the pathophysiological processes underlying the disease, intervention programs, patient focused education, and self management protocols.

\section{Dovepress}

This journal is indexed on PubMed Central, MedLine and CAS. The manuscript management system is completely online and includes a very quick and fair peer-review system, which is all easy to use. Visit http://www.dovepress.com/testimonials.php to read real quotes from published authors.

Submit your manuscript here: http://www.dovepress.com/international-journal-of-chronic-obstructive-pulmonary-disease-journal 